\title{
Autophagy dysfunction upregulates beta-amyloid peptides via enhancing the activity of $\gamma$-secretase complex
}

This article was published in the following Dove Press journal:

Neuropsychiatric Disease and Treatment

17 August 2015

Number of times this article has been viewed

\section{Zhiyou Cai' \\ Yingjun Zhou' \\ Zhou Liu ${ }^{2,3}$ \\ Zunyu Ke \\ Bin Zhao 2,3}

'Department of Neurology, Renmin Hospital, Hubei University of Medicine, Shiyan, Hubei Province, ${ }^{2}$ Department of Neurology, The Affiliated Hospital of Guangdong Medical College, ${ }^{3}$ Institute of Neurology, Guangdong Medical College, Zhanjiang, Guangdong Province, People's Republic of China

Correspondence: Zhiyou Cai Department of Neurology, Renmin Hospital, Shiyan Renmin Hospital, Hubei University of Medicine, Chaoyang 39, Maojian District, Shiyan, Hubei Province 442000, People's Republic of China

Tel +86 7I9 8637909

Fax+867198637909

Email c0909@hotmail.com

Bin Zhao

Department of Neurology, The Affiliated Hospital of Guangdong Medical College, No 2 Wenming Dong Street, Xiashan District, Zhanjiang, Guangdong Province 524023, People's Republic of China

Tel +86759238 8505

Fax +867592284104

Email zhaobingmc@I63.com
Abstract: Numerous studies have shown that autophagy failure plays a critical role in the pathogenesis of Alzheimer's disease, including increased expression of beta-amyloid (A $\beta$ ) protein and the dysfunction of $\mathrm{A} \beta$ clearance. To further evaluate the role of autophagy in Alzheimer's disease, the present study was implemented to investigate the effects of autophagy on $\alpha$-secretase, $\beta$-secretase, or $\gamma$-secretase, and observe the effects of autophagy on autophagic clearance markers. These results showed that both autophagy inhibitor and inducer enhanced the activity of $\alpha-, \beta$-, and $\gamma$-secretases, and $\mathrm{A} \beta$ production. Autophagy inhibitor may more activate $\gamma$-secretase and promote $\mathrm{A} \beta$ production and accumulation than its inducer. Both autophagy inhibitor and inducer had no influence on $A \beta$ clearance. Hence, autophagy inhibitor may activate $\gamma$-secretase and promote $\mathrm{A} \beta$ production and accumulation, but has no influence on $\mathrm{A} \beta$ clearance.

Keywords: Alzheimer's disease, autophagy, beta-amyloid, secretases

\section{Introduction}

Alzheimer's disease (AD) is a progressive neurodegenerative disease, of which cognitive impairment gradually worsens over time..$^{1-3} \mathrm{As} \mathrm{AD}$ advances through the brain, it eventually affects all aspects of a person's life including mental abilities, emotions and moods, behavior, and the ability to carry out daily activities like eating and grooming. ${ }^{4,5}$ It is well known that there are three consistent neuro-pathological hallmarks associated with $\mathrm{AD}$, including extracellular amyloid-rich senile plaques, intracellular neurofibrillary tangles and neuronal degeneration of basal forebrain cholinergic neurons that innervate the hippocampus and the cortex. ${ }^{6-8}$

Autophagy is one of the major degradation pathway characterized by a ubiquitous cellular process responsible for the bulk degradation of long-lived proteins and organelles through an autophagosome-lysosomal pathway. ${ }^{9,10}$ The word autophagy is derived from the Greek words auto (meaning "self") and phage (meaning "eat"), of which the main function of autophagy refers to clear abnormal or obsolete cellular proteins. ${ }^{11}$ There are at least three processes by which intracellular constituents enter lysosomes for degradation distinguishable by their mechanisms: macro-autophagy (the most prevalent form), micro-autophagy, and chaperone-mediated autophagy. Autophagy exists in both normal cellular homeostasis and disease states. Increasing findings have demonstrated that autophagosome-lysosomal dysfunction contributes to severe neurodegenerative disorders related to accumulations of lysosomes and autophagic vacuoles (AVs). ${ }^{11}$ Compelling research studies have supported that the pivotal role of autophagy in the clearance of aggregate-prone proteins is responsible for several neurodegenerative disorders, ${ }^{12,13}$ which are implicated in the pathogenesis 
of AD, ${ }^{13,14}$ Parkinson's disease,${ }^{15,16}$ Huntington's disease, ${ }^{17,18}$ and other related disorders.

An autophagosome, a spherical structure with double layer membranes, is a cellular vesicle that ingests cellular debris and transports the debris to lysosomes. Growing evidence indicates that the rate of autophagosome formation and maturation and the efficiency of autophagosome/ lysosome fusion decline in neurodegenerative diseases with age. ${ }^{19-21}$ A growing number of studies have shown that dysfunction of autophagy plays a critical role in the pathogenesis of AD, including senile plaques, neurofibrillary tangles, and neuronal degeneration. ${ }^{13,22}$ Moreover, it has been found that immature AVs accumulate during the early evolution of pathology in a dendrite in the PS1-APP (amyloid protein precursor) mouse model of AD while pathological AVs' accumulation is associated with inhibited retrograde AVs' transport and impaired autophagosome/lysosome fusion. ${ }^{23-25}$ Furthermore, a link between autophagy dysfunction and beta-amyloid $(\mathrm{A} \beta)$ generation and clearance has been reported to occur in $\mathrm{AD} \cdot{ }^{26,27} \mathrm{~A}$ number of papers have investigated the precise role of autophagy in the $A \beta$ generation and clearance. However, understanding the exact mechanism may help to design more effective therapeutic strategies to prevent neuronal degeneration and death. It is well known that $\mathrm{A} \beta$ production and deposition represent a key feature and is thought as the classic pathological hallmarks in AD. $A \beta$ is generated from APP by the sequential actions of two proteolytic enzymes: $\beta$-secretase (beta-site APP cleavage enzyme, BACE) and $\gamma$-secretase complex. ${ }^{28,29}$ In addition, APP undergoes another cleavage: the non-amyloidogenic processing by $\alpha$-secretase and $\gamma$-secretase complex to release membrane-anchored carboxy-terminal fragments that may be associated with apoptosis. ${ }^{30,31}$ Therefore, it is possible that autophagy regulated $\mathrm{A} \beta$ generation via controlling the activity of $\alpha$-, $\beta$-, or $\gamma$-secretases. The present study was implemented to investigate the effects of autophagy on $\alpha-$, $\beta$-, and $\gamma$-secretase, and the level of $A \beta$, and to observe the effects of autophagy on autophagic clearance markers. The aim is to further evaluate the role of autophagy in the neurodegenerative process of AD.

These results noted that the both autophagy inhibitor and inducer enhanced $A \beta 1-42$ expression while the level of $A \beta 1-42$ peptide was more remarkably increased by the autophagy inhibitor than by the autophagy inducer. Both autophagy inhibitor and inducer increased the activity of $\alpha-, \beta$-, and $\gamma$-secretases while the components of the $\gamma$-secretase complex (Presenilin 1, Nicastrin, and presenilin enhancer 2 [Pen-2]) were more activated by autophagy inhibitor, compared with the inducer treatment. However, this study revealed that there was no difference between the treatment of the autophagy inhibitor and autophagy inducer. Our study suggests that autophagy inhibitor may activate $\gamma$-secretase and promote $A \beta$ accumulation, but has no influence on $\mathrm{A} \beta$ clearance.

\section{Materials and methods Cell culture}

SH-SY5Y, a human-derived neuroblastoma cell line, is thrice-cloned originally from SK-N-SH and widely used in the scientific research of neurodegenerative disorders. ${ }^{32}$ SH-SY5Y was grown in Dulbecco's Modified Eagle's Medium (Thermo Fisher Scientific, Waltham, MA, USA) supplemented with 10\% fetal bovine serum (HyClone, Logan, UT, USA) and 1\% penicillin/streptomycin. Cells were maintained in a humidified atmosphere at $37^{\circ} \mathrm{C}$ with $5 \% \mathrm{CO}_{2}$. The autophagy inhibitor (3-methyladenine, 3-MA, Santa Cruz Biotechnology, Dallas, TX, USA) and inducer (STF-62247, Selleck Chemicals, Houston, TX, USA) were dissolved in dimethyl sulfoxide and used at the following concentrations: the inhibitor (3-MA), $10 \mathrm{mM}$; the inducer (STF-62247), $10 \mu \mathrm{M}$. The cells were treated with the autophagy inhibitor and inducer without fetal bovine serum for 24 hours.

\section{RNA extraction and real-time quantitative PCR}

Total cellular RNA was extracted using the Trizol reagent (Invitrogen), according to the manufacturer's instruction. cDNAs were synthesized and real-time PCR was performed using the GoTaq ${ }^{\circledR}$ 2-Step RT-qPCR System (Promega, Madison, WI, USA) in an ABI Prism 7500 Sequence Detection System (Applied Biosystems, Foster City, CA, USA). Gene expression data were normalized to the geometric mean of the $\beta$-actin, housekeeping gene, to control for variability

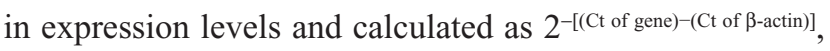
where $\mathrm{Ct}$ represents the threshold cycle for each transcript. The used primers in this study were the following: BACE1 (forward: 5'-AGGCAGCTGTCCAGCACATACC-3', reverse: 3'-TAGCCAGCTGGTGCAGGAGAT-5'), a disintegrin and metalloprotease 17 (ADAM17) (forward: 5'-AGCAGGTGTCGTTGTTCAGA-3', reverse: 3'-GTGGCGATCACGAGAACAAT-5'), Presenilin 1 (forward: 5'-CAGGTGCTATAAGGTCATCC-3', reverse: 3'-GTCCACAGCAACGTTATAGG-5'), Presenilin 2 (forward: 5'-CAGCTCATCTACACGCCATT-3', reverse: 3'-CAGCAGCATCAGTGAAGACA-5'), Nicastrin (forward: 5'-TACGGAACCAGGTGGAGGAT-3', reverse: 
3'-GAAGGCACCAGAGTGGTCAG-5'), APH-1 (forward: 5'-TCCTGACTTCAGCCTTTCTGAC-3', reverse: 3'-CAAGAGGCTGCGCTGAATAC-5'), Pen-2 (forward: 5'-GCCAAATCAAAGGCTATGTCTG-3', reverse: 3'-ATGGTGAAGGAGAGGTAGTC-5'), $\beta$-actin (forward: 5'-TGGCACCCAGCACAATGAA-3', reverse: $3^{\prime}$-CTAAG TCATAGTCCGCCTAGAAGCA-5').

\section{Western blotting}

Cells were harvested in sampling buffer $(62.5 \mathrm{mmol} / \mathrm{L}$ Tris-HCl [pH 6.8], 10\% glycerol, 2\% SDS) and heated for 5 minutes at $100^{\circ} \mathrm{C}$. The concentration of extracted proteins was determined by the Bradford assay using a commercial kit (Bio-Rad, Berkeley, CA, USA). Equal quantities of protein were separated by electrophoresis on $12 \%$ SDS/ polyacrylamide gels and transferred onto polyvinylidene difluoride membranes (Roche, Indianapolis, IN, USA). The membranes were then probed with rabbit primary antibodies against anti-BACE1, anti-ADAM17, anti-Presenilin 1, anti-Presenilin 2, anti-Nicastrin, anti-APH-1, anti-Pen-2, neprilysin (NEP), insulin-degrading enzyme (IDE), endothelin-converting enzyme 1 (ECE-1), and ECE-2 (1:1,000; Santa Cruz Biotechnology). The protein expression was detected with horseradish peroxidase-conjugated goat anti-rabbit IgG (1:2,000; Amersham Pharmacia Biotech, Piscataway, NJ, USA) and an enhanced chemiluminescence kit (Amersham Pharmacia Biotech) according to the manufacturer's instructions. Anti- $\beta$-actin mouse monoclonal antibody $(1: 1,000$; Cell Signaling, Danvers, MA, USA) acted as a loading control.

\section{Transmission electron microscopy}

SH-SY5Y treated with 3-MA and STF-62247 for 24 hours was fixed for 2 hours at room temperature with $2.5 \%$ glutaraldehyde in phosphate-buffered saline $(\mathrm{pH} \mathrm{7.4)}$ and, subsequently, with $1 \% \mathrm{OsO}_{4}$ in $50 \mathrm{mM}$ sodium cacodylate buffer ( $\mathrm{pH} 7.3$ ), dehydrated in an ethanol series and embedded into epon (catalyst). Ultrathin sections of $50 \mathrm{~nm}$ were contrasted with uranyl acetate and lead citrate and analyzed in a Tecnai Spirit transmission electron microscope (FEI) with an ORIUS CCD camera (Gatan).

\section{A $\beta I-42$ measurement}

Cells were seed at a constant density to obtain identical experimental conditions in the different tests and to achieve a high accuracy of the measurements. A $\beta 1-42$ levels were determined in the culture supernatant using an ELISA kit (Uscn Life). The assays were performed according to manufacturer's guidelines. Results were expressed as $\mathrm{pg} / \mathrm{mL}$.

\section{Statistical analysis}

Student's $t$-test was used to evaluate the significant difference between two groups of data in all the pertinent experiments. Data were represented as the mean \pm standard error of the mean. $P$-value $<0.05$ (using a two-tailed paired $t$-test) was considered as statistically significant.

\section{Ethics}

This study was approved by the Ethics committee of Renmin Hospital, Hubei University of Medicine.

\section{Results}

\section{Inhibiting autophagy pathway increased $A \beta$ expression}

Mounting hypothesis has shown that extracellular $A \beta$ plays an important role in $\mathrm{AD}$ pathogenesis. ${ }^{22,33,34}$ Consistent with other research results, the level of A $1-42$ peptide was remarkably increased by the autophagy inhibitor (3-MA), compared with control and the autophagy inducer (STF62247) $(P<0.001)$ (Figure 1). Different from the other research results, both the autophagy inhibitor (3-MA) and inducer (STF-62247) enhanced A $\beta 1-42$ expression whereas the level of $A \beta 1-42$ stimulated by 3-MA was higher than that by STF-62247 $(P<0.005)$ (Figure 1).

\section{Autophagy dysfunction augmented the activity of $\gamma$-secretase complex}

APP is cleaved sequentially at the extracellular site by $\beta$-secretase (BACE) and $\gamma$-secretase to release A $\beta$ peptides. ${ }^{35-37}$ APP can be also cleaved by $\alpha$-and $\gamma$-secretases to produce P3. The $\alpha$-secretase represents two members of the family of ADAM: tumor necrosis factor-converting enzyme (ADAM17) and ADAM10. $\beta$-Secretase, a membrane-bound aspartic protease, is also called BACE. ${ }^{36,38} \gamma$-Secretase is a multi-subunit protease complex, consisting of four individual proteins: Presenilin, Nicastrin, APH-1 (anterior pharynxdefective 1), and Pen-2. ${ }^{35,38}$

In the present study, the glioma cell line SH-SY5Y was treated with autophagy inhibitor (3-MA) and autophagy inducer (STF-62247). Then, the cell harvested to analyze by real-time quantitative PCR and Western blotting. These results demonstrated that $\alpha$ - and $\beta$-secretases, respectively exhibited as BACE1 and ADAM17, were not different between 3-MA and STF-62247 treatments. The components of the $\gamma$-secretase complex (Presenilin 1, Nicastrin, and Pen-2) were activated by 3-MA, compared with the STF62247 treatment $(P<0.001)$ (Figure 2$)$. Both autophagy inhibitor and inducer improved the activity of $\alpha$-, $\beta$-, and $\gamma$-secretases $(P<0.005)$. 

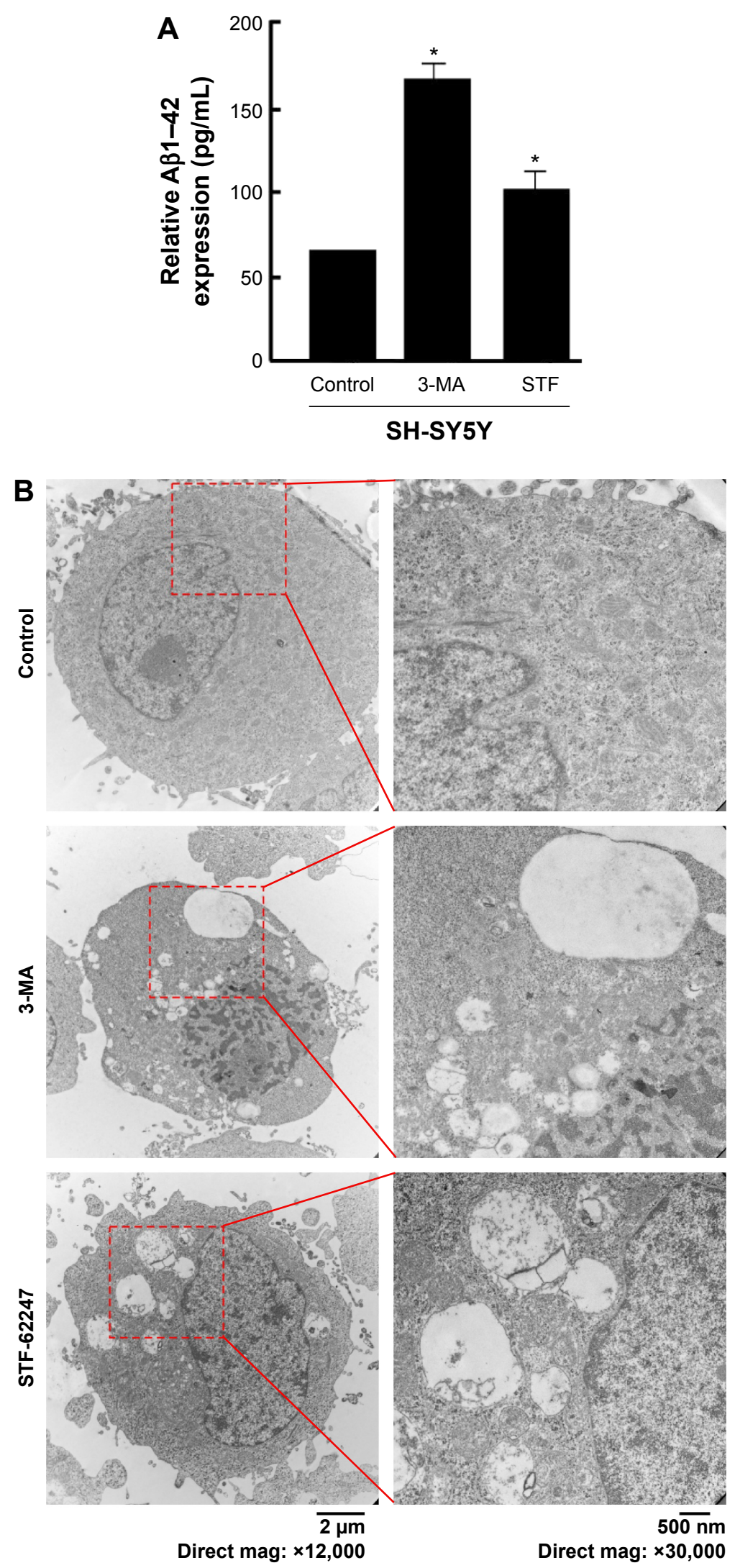

Figure I Modulation of autophagy affects $A \beta$ secretion.

Notes: The autophagy inhibitor (3-MA) significantly enhanced A $\beta$ expression in the neuroblastoma SH-SY5Y cell line, compared with the control and autophagy inducer (STF62247) $(* P<0.00 I$ ). In addition, the autophagy inducer (STF-62247) also increased the level of $A \beta I-42$ peptide (in supernatant) (*P<0.005). The level of $A \beta I-42$ stimulated by $3-M A$ was higher than the level by STF-62247 $(* P<0.005)(\mathbf{A})$. The result by transmission electron microscopy showed that 3-MA prevented the formation of autolysosome by inhibiting autophagosome formation while STF-62247 enhanced the combination between autophagosome and lysosome to benefit the autophagosome fusion and breakdown (B).

Abbreviations: $A \beta$, beta-amyloid; 3-MA, 3-methyladenine; mag, magnification. 

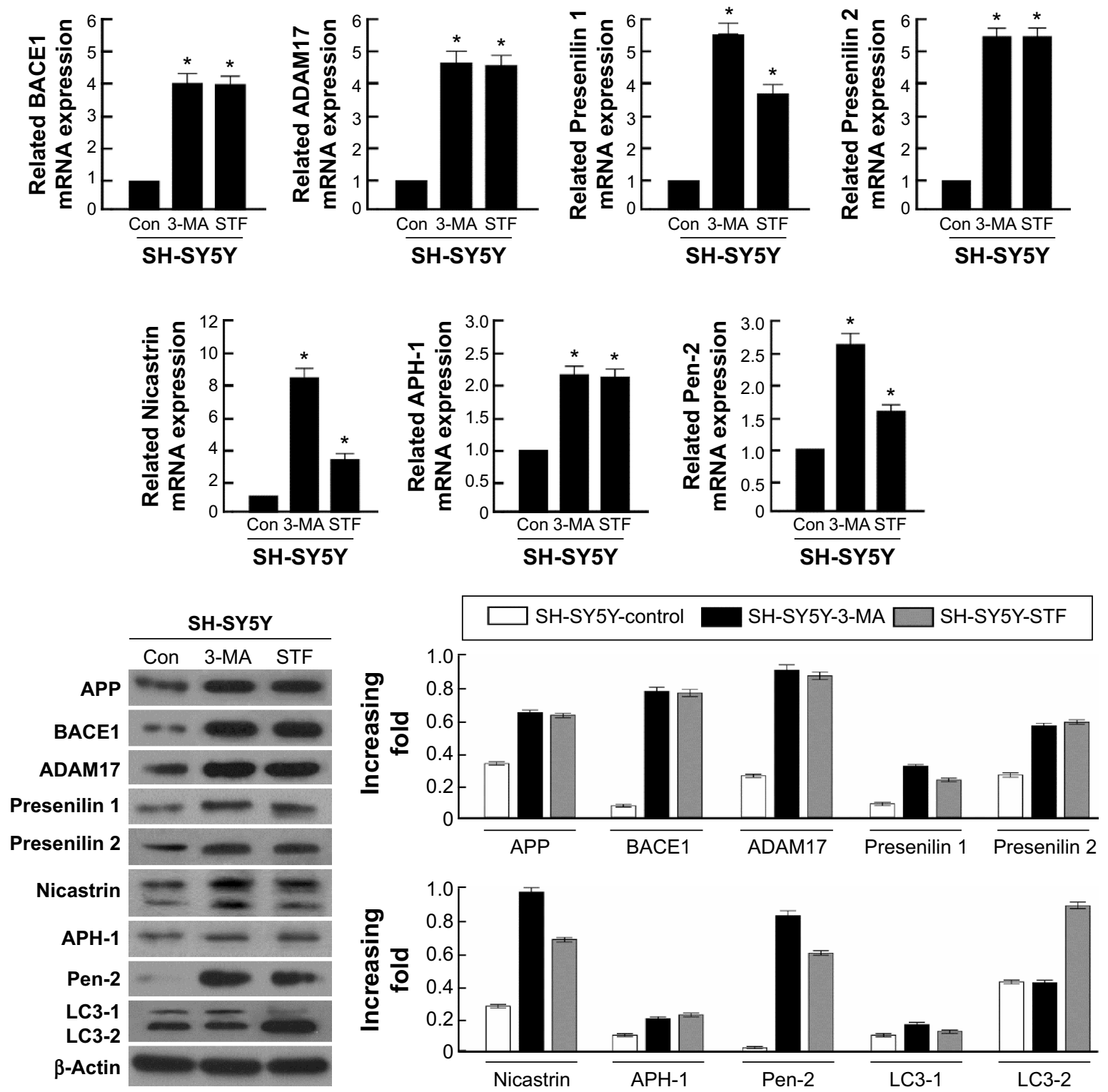

Figure 2 Effect of autophagy on APP-related cleavage secretases.

Notes: Columns depict relative densitometric values that were obtained by comparing with $\beta$-actin. Presenilin I, Nicastrin, and Pen- 2 were increased with the 3-MA treatment $(* P<0.00 \mathrm{I})$ while the other component of the $\gamma$-secretase complex $(\mathrm{APH}-\mathrm{I}$ and Presenilin 2$)$ has no difference, compared with the STF-62247 treatment. Moreover, BACEI and ADAMI7 respectively had no difference between 3-MA and STF- 62247 treatments. Both autophagy inhibitor and inducer activated $\alpha$-, $\beta$-, and $\gamma$-secretases $(* P<0.005)$. Abbreviations: A 3 , beta-amyloid; ADAMI7, a disintegrin and metalloprotease 17; APH-I, anterior pharynx-defective I; APP, amyloid protein precursor; BACEI, beta-site APP cleavage enzyme; 3-MA, 3-methyladenine; Con, control, LC3, microtubule-associated protein IA/IB-light chain 3.

\section{Autophagy had no effect on the $A \beta$ clearance markers}

$A \beta$ production and failure of $A \beta$ clearance are key factors in the development of $\mathrm{AD}$. An overall impairment in $\mathrm{A} \beta$ clearance has been found where clearance rates for both $A \beta 1-42$ and $\mathrm{A} \beta 1-40$ were impaired in AD. ${ }^{35,39}$ Several A $\beta$-degrading enzymes such as NEP, IDE, ECE-1, and ECE-2 are critical in $A \beta$ accumulation determined in part by the imbalance between the production of $A \beta$ and its removal from the brain..$^{35,40,41}$ Therefore, the present study further investigated the influence of autophagy on the $A \beta$ clearance markers
(NEP, IDE, ECE-1, and ECE-2) although several research supported that the activation of autophagy benefited $A \beta$ clearance. However, these findings revealed that there was no difference between the treatment of the autophagy inhibitor (3-MA) and autophagy inducer (STF-62247) $(P>0.05)$ (Figure 3).

\section{Discussion}

Autophagy is one major cellular pathway associated with the removal of aggregated proteins. A few studies elucidated that autophagy plays a critical role in multiple pathological 

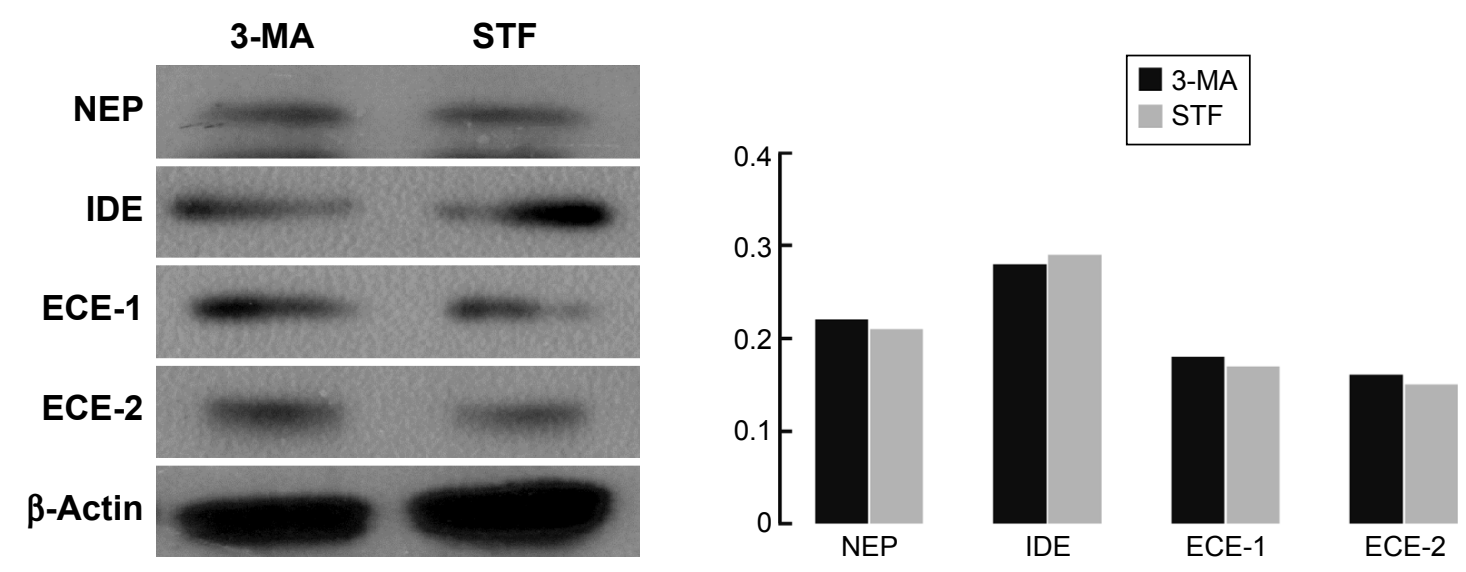

Figure 3 Influence of autophagy on the $A \beta$ clearance markers.

Notes: Columns depict relative densitometric values that were obtained by comparing with $\beta$-actin. There was no change in the level of NEP, IDE, ECE-I, and ECE-2 by the treatment of the autophagy inhibitor (3-MA) and autophagy inducer (STF-62247) $(P>0.05)$ on the SH-SY5Y cell line.

Abbreviations: A $\beta$, beta-amyloid; ECE-I, endothelin-converting enzyme I; ECE-2, endothelin-converting enzyme 2; IDE, insulin-degrading enzyme; NEP, neprilysin; 3-MA, 3-methyladenine.

lesions of AD, such as dysregulating APP turnover and enhancing the activity of $\beta$ - and/or $\gamma$-secretases. ${ }^{42-44}$ Autophagy affects an array of molecular pathways that may play a role in both $A \beta$ generation and $A \beta$ clearance. ${ }^{22,45,46}$ However, the precise mechanism or role of autophagy in $A \beta$ generation and $A \beta$ clearance remained unclear. ${ }^{22}$ We found that both autophagy inhibitor and inducer are associated with $\mathrm{A} \beta$ generation via regulating $\alpha-, \beta-$, and/or $\gamma$-secretases. The relationship between autophagy inhibitor and the level of $A \beta$ is robust enough for autophagy inducer, which heightened $A \beta$ expression through upregulating $\alpha-, \beta-$, and/or $\gamma$-secretases. Nevertheless, there was no divergence in $A \beta$ clearance under the treatment of autophagy inhibitor and inducer.

Our study has certain distinctive outcome. Formerly, inducing autophagy pathway keeps a foothold to restrain $A \beta$ generation whereas inhibiting autophagy pathway is a friend for $\mathrm{A} \beta$ generation. Yet, the present results were different from the past whereas this study illustrated that both autophagy inhibitor and inducer account for $A \beta$ generation. Consistent with the former findings, inhibiting autophagy pathway was a more strongly enhancer to the event of $A \beta$ generation. Thus, it seems that our results were self-contradictory and paradox. It may be reasonable to take into consideration that $A \beta$ plays a dual role as both physical and pathological substance. Functional A $\beta$ is abundant in most environmental biofilms. Several potential activities have been discovered for $A \beta$, such as activating kinase enzymes, protection against oxidative stress, regulation of cholesterol transport, functioning as a transcription factor, and anti-microbial activity. ${ }^{47-50}$ Intracellular $A \beta$ may impel a variety of cellular events such as protein degradation, axonal transport, neuronal firing, and autophagy to apoptosis. ${ }^{47,51-53}$ Therefore, it is indicated that autophagy plays dual roles in the degradation and secretion of $A \beta$ since inducing and inhibiting autophagy pathway shares a common access to heighten $A \beta$ production under the $A \beta$ physical process (Figure 4).

It is well known that $A \beta$ is generated from APP by the sequential cleavage of two proteolytic enzymes: $\beta$-(BACE) and $\gamma$-secretase. ${ }^{54,55} \mathrm{~A}$ few studies demonstrated that the role of autophagy has been linked to $A \beta$ generation and aberrant processing of APP. ${ }^{56,57}$ Autophagy plays an important role in $A \beta$ generation via regulating APP turnover and stimulating the activity of $\beta$ - and $\gamma$-secretases. ${ }^{58,59}$ However, the key role of autophagy in AD development is still under consideration today ${ }^{60}$ Whether autophagy regulates $A \beta$ by influencing the expression of these associated secretases $(\alpha-, \beta-$, and/or $\gamma$-secretases)? In controversy with the other findings which indicate that autophagy inducer can inhibit the expression of $\beta$ - and $\gamma$-secretases, and $A \beta$ generation, it was occurred that both autophagy inhibitor and inducer increased the activity of $\alpha-, \beta$-, and $\gamma$-secretases in the present study. In consideration to the physiological function of APP, $\alpha-, \beta$-, and $\gamma$-secretases, ${ }^{49,61}$ it may be a normal response to the stimulation of autophagy inducer and acute stress.

Importantly, this study demonstrated that autophagy inhibitor augmented the activity of $\gamma$-secretase complex by upregulating its components (Presenilin 1, Nicastrin, and Pen-2), compared with autophagy inducer. Accordingly, it was inferred that autophagy failure contributed to $A \beta$ generation via activating $\gamma$-secretase complex (Figure 4). 


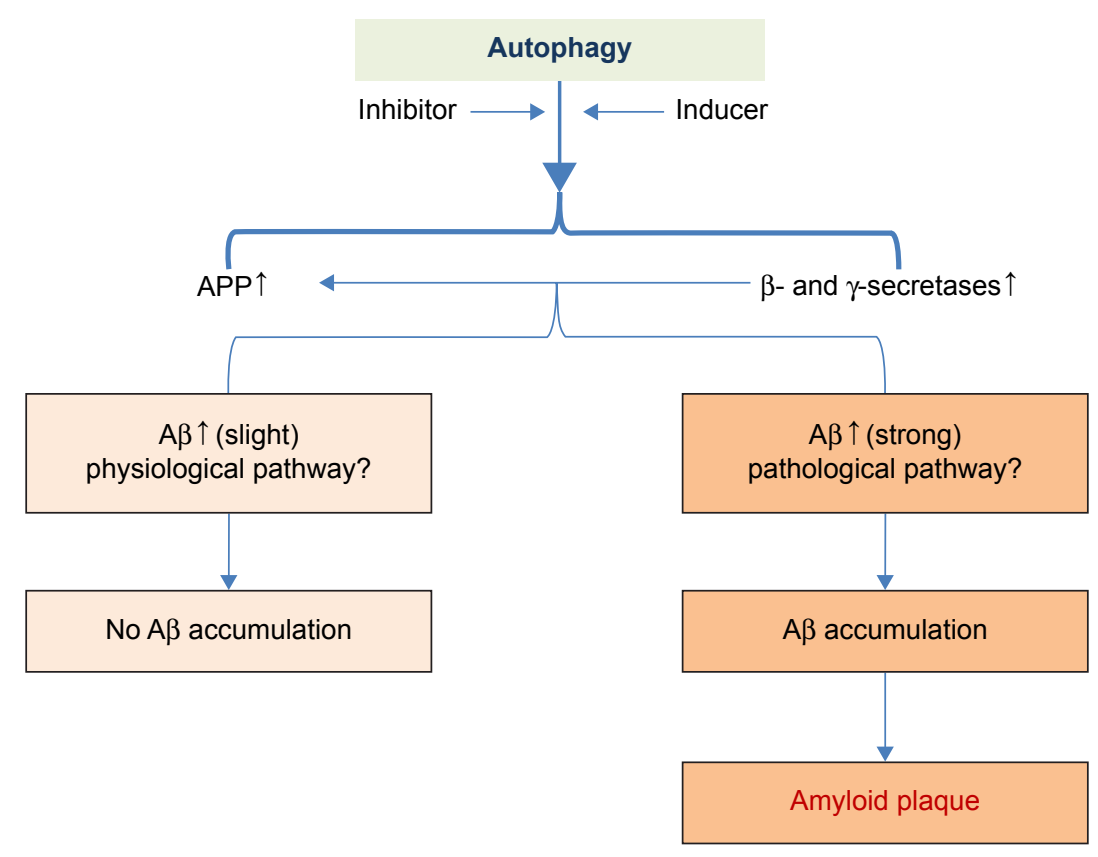

Figure 4 The hypothesis of the role of autophagy in the $A \beta$ pathophysiology process.

Notes: $A \beta$ may play a dual role as both physical and pathological substance. Functional $A \beta$ is abundant in most environmental biofilms, such as activating kinase enzymes, protection against oxidative stress, regulating cholesterol transport, functioning as a transcription factor, and anti-microbial activity. Intracellular A $\beta$ may impel a variety of cellular events such as protein degradation, axonal transport, neuronal firing, and autophagy to apoptosis. Therefore, it is indicated that autophagy plays dual roles in the degradation and secretion of $A \beta$.

Abbreviations: $A \beta$, beta-amyloid; APP, amyloid protein precursor.

Several studies have supported that failure of $A \beta$ clearance in the brains of patients with $\mathrm{AD}$ has been involved in autophagy dysfunction. ${ }^{62,63}$ The $\mathrm{A} \beta$ deposition and formation of $\mathrm{A} \beta$ plaques will be accelerated because of defects in its removal, mediated through a combination of diffusion along perivascular extracellular matrix, transport across vessel walls into the blood stream, and enzymatic degradation. ${ }^{44,64}{ }^{66}$ Several A $\beta$-degrading enzymes such as NEP, IDE, ECE-1, and ECE-2 are critical in $\mathrm{A} \beta$ clearance, most of which are produced by neurons and glial cells. ${ }^{40,41}$ Scientific evidence has revealed the role of autophagy in $\mathrm{A} \beta$ clearance involved in $\mathrm{A} \beta$-degrading enzymes. ${ }^{40,44}$ However, the clearance markers of $A \beta$ (NEP, IDE, ECE-1, and ECE-2) were not different between autophagy inducer and inhibitor treatment. These results showed that, autophagy inhibitor may activate $\gamma$-secretase and promote $A \beta$ generation and accumulation, but has no influence on $\mathrm{A} \beta$ clearance.

In the current study, both autophagy inhibitor and inducer increased the activity of $\alpha$-, $\beta$-, and $\gamma$-secretases, and enhanced $\mathrm{A} \beta$ production. Moreover, autophagy inhibitor may more activate $\gamma$-secretase and promote $A \beta$ production and accumulation, compared with its inducer. Both autophagy inhibitor and inducer had no influence on $A \beta$ clearance. However, other results indicate that inducing autophagy pathway keeps a foothold to restrain $A \beta$ generation whereas inhibiting autophagy pathway is a friend for $A \beta$ generation. Aberrant autophagy induction leads into a concentration of AVs rich in APP, A $\beta$ and the elements crucial for its formation while the dysfunction of autophagic clearance plays an important role in $\mathrm{AD}$ pathogenesis. ${ }^{60}$ Overall, the precise role of in $\mathrm{AD}$ pathogenesis is still under contention. ${ }^{11}$ Therefore, it is indispensable to further elucidate the potential routes for autophagy-mediated $\mathrm{A} \beta$ production and clearance in the $\mathrm{AD}$ pathophysiology mechanisms.

\section{Acknowledgments}

This study was supported by the National Nature Science Foundation of China (81070878/H0902) and Nature Science Foundation of Guangdong (S20120200-10867) to Professor Bin Zhao, the Hubei Province Health and Family Planning Scientific Research Project (WJ2015MB219), the Hubei Provincial Nature Science Foundation (2015CFB260), and the Shiyan Nature Science Foundation (15K70) to Dr Zhiyou Cai.

\section{Disclosure}

The authors report no conflicts of interest in this work.

\section{References}

1. Mhatre SD, Michelson SJ, Gomes J, Tabb LP, Saunders AJ, Marenda DR Development and characterization of an aged onset model of Alzheimer's disease in Drosophila melanogaster. Exp Neurol. 2014; 261C:772-781. 
2. Fargo K, Bleiler L. Alzheimer's Association report. Alzheimers Dement. 2014;10:e47-e92.

3. Choi SJ, Jung SS, You YS, et al. Prevalence of Alzheimer's dementia and its risk factors in community-dwelling elderly Koreans. Psychiatry Investig. 2008;5:78-85.

4. Hsieh S, Hodges JR, Piguet O. Recognition of positive vocalizations is impaired in behavioral-variant frontotemporal dementia. $J$ Int Neuropsychol Soc. 2013;19:483-487.

5. Teri L, Logsdon R, Yesavage J. Measuring behavior, mood, and psychiatric symptoms in Alzheimer disease. Alzheimer Dis Assoc Disord. 1997; 11(Suppl 6):50-59.

6. Hartig W, Kacza J, Paulke BR, et al. In vivo labelling of hippocampal beta-amyloid in triple-transgenic mice with a fluorescent acetylcholinesterase inhibitor released from nanoparticles. Eur J Neurosci. 2010;31: 99-109.

7. Heese K, Akatsu H. Alzheimer's disease - an interactive perspective. Curr Alzheimer Res. 2006;3:109-121.

8. da Cruz e Silva OA, Henriques AG, Domingues SC, da Cruz e Silva EF. Wnt signalling is a relevant pathway contributing to amyloid betapeptide-mediated neuropathology in Alzheimer's disease. CNS Neurol Disord Drug Targets. 2010;9:720-726.

9. Melendez A, Levine B. Autophagy in C. elegans. Pasadena (CA): Worm Book; 2009:1-26.

10. Chaumorcel M, Souquere S, Pierron G, Codogno P, Esclatine A. Human cytomegalovirus controls a new autophagy-dependent cellular antiviral defense mechanism. Autophagy. 2008;4:46-53.

11. Funderburk SF, Marcellino BK, Yue Z. Cell "self-eating” (autophagy) mechanism in Alzheimer's disease. Mt Sinai J Med. 2010;77:59-68.

12. Zhou X, Yang C, Liu Y, et al. Lipid rafts participate in aberrant degradative autophagic-lysosomal pathway of amyloid-beta peptide in Alzheimer's disease. Neural Regen Res. 2014;9:92-100.

13. Yang Y, Chen S, Zhang J, et al. Stimulation of autophagy prevents amyloid-beta peptide-induced neuritic degeneration in PC12 cells. J Alzheimers Dis. 2014;40:929-939.

14. Moreira PI, Siedlak SL, Wang X, et al. Increased autophagic degradation of mitochondria in Alzheimer disease. Autophagy. 2007;3: 614-615.

15. Arduino DM, Esteves AR, Cardoso SM. Mitochondria drive autophagy pathology via microtubule disassembly: a new hypothesis for Parkinson disease. Autophagy. 2013;9:112-114.

16. Yang Q, Mao Z. Parkinson disease: a role for autophagy? Neuroscientist. 2010;16:335-341.

17. Koga H, Martinez-Vicente M, Arias E, Kaushik S, Sulzer D, Cuervo AM. Constitutive upregulation of chaperone-mediated autophagy in Huntington's disease. J Neurosci. 2011;31:18492-18505.

18. Martinez-Vicente M, Talloczy Z, Wong E, et al. Cargo recognition failure is responsible for inefficient autophagy in Huntington's disease. Nat Neurosci. 2010;13:567-576.

19. Wong YC, Holzbaur EL. The regulation of autophagosome dynamics by huntingtin and HAP1 is disrupted by expression of mutant huntingtin, leading to defective cargo degradation. J Neurosci. 2014;34: 1293-1305.

20. Rajawat YS, Hilioti Z, Bossis I. Aging: central role for autophagy and the lysosomal degradative system. Ageing Res Rev. 2009;8:199-213.

21. Tan CC, Yu JT, Tan MS, Jiang T, Zhu XC, Tan L. Autophagy in aging and neurodegenerative diseases: implications for pathogenesis and therapy. Neurobiol Aging. 2014;35:941-957.

22. Nixon RA. Alzheimer neurodegeneration, autophagy, and Abeta secretion: the ins and outs (comment on DOI:10.1002/bies.201400002). Bioessays. 2014;36:547.

23. Li L, Zhang S, Zhang X, et al. Autophagy enhancer carbamazepine alleviates memory deficits and cerebral amyloid-beta pathology in a mouse model of Alzheimer's disease. Curr Alzheimer Res. 2013;10: 433-441.

24. Yu WH, Cuervo AM, Kumar A, et al. Macroautophagy - a novel Betaamyloid peptide-generating pathway activated in Alzheimer's disease. J Cell Biol. 2005;171:87-98.
25. Lee JK, Jin HK, Park MH, et al. Acid sphingomyelinase modulates the autophagic process by controlling lysosomal biogenesis in Alzheimer's disease. J Exp Med. 2014;211:1551-1570.

26. Wolfe DM, Lee JH, Kumar A, Lee S, Orenstein SJ, Nixon RA. Autophagy failure in Alzheimer's disease and the role of defective lysosomal acidification. Eur J Neurosci. 2013;37:1949-1961.

27. Nixon RA. Autophagy, amyloidogenesis and Alzheimer disease. JCell Sci. 2007;120:4081-4091.

28. Allsop D, Yamamoto T, Kametani F, Miyazaki N, Ishii T. Alzheimer amyloid beta/A4 peptide binding sites and a possible 'APP-secretase' activity associated with rat brain cortical membranes. Brain Res. 1991; 551:1-9.

29. Hata S, Fujishige S, Araki Y, et al. Alcadein cleavages by amyloid beta-precursor protein (APP) alpha- and gamma-secretases generate small peptides, p3-Alcs, indicating Alzheimer disease-related gammasecretase dysfunction. J Biol Chem. 2009;284:36024-36033.

30. Deng $Y$, Wang $Z$, Wang $R$, et al. Amyloid- $\beta$ protein $(A \beta)$ Glu11 is the major $\beta$-secretase site of $\beta$-site amyloid- $\beta$ precursor proteincleaving enzyme 1 (BACE1), and shifting the cleavage site to $\mathrm{A} \beta$ Asp1 contributes to Alzheimer pathogenesis. Eur J Neurosci. 2013;37: 1962-1969.

31. Fahrenholz F. Alpha-secretase as a therapeutic target. Curr Alzheimer Res. 2007;4:412-417.

32. Cai Z, Li B, Li K, Zhao B. Down-regulation of amyloid- $\beta$ through AMPK activation by inhibitors of GSK-3 $\beta$ in SH-SY5Y and SH-SY5YAßPP695 cells. J Alzheimers Dis. 2012;29:89-98.

33. Fernandez MA, Klutkowski JA, Freret T, Wolfe MS. Alzheimer presenilin-1 mutations dramatically reduce trimming of long amyloid $\beta$-peptides $(\mathrm{A} \beta$ ) by gamma-secretase to increase 42 -to-40-residue $\mathrm{A} \beta$. J Biol Chem. 2014;289(45):31043-31052.

34. Swomley AM, Forster S, Keeney JT, et al. Abeta, oxidative stress in Alzheimer disease: evidence based on proteomics studies. Biochim Biophys Acta. 2014;1842:1248-1257.

35. Cai Z, Zhao B, Li K, et al. Mammalian target of rapamycin: a valid therapeutic target through the autophagy pathway for Alzheimer's disease? J Neurosci Res. 2012;90:1105-1118.

36. Cai Z, Zhao B, Ratka A. Oxidative stress and beta-amyloid protein in Alzheimer's disease. Neuromolecular Med. 2011;13:223-250.

37. Cai Z, Yan LJ, Li K, Quazi SH, Zhao B. Roles of AMP-activated protein kinase in Alzheimer's disease. Neuromolecular Med. 2012;14:1-14.

38. Cai Z, Zhao Y, Zhao B. Roles of glycogen synthase kinase 3 in Alzheimer's disease. Curr Alzheimer Res. 2012;9:864-879.

39. Mawuenyega KG, Sigurdson W, Ovod V, et al. Decreased clearance of CNS beta-amyloid in Alzheimer's disease. Science. 2010;330:1774.

40. Cai Z, Hussain MD, Yan LJ. Microglia, neuroinflammation, and betaamyloid protein in Alzheimer's disease. Int J Neurosci. 2014;124: 307-321.

41. Yan LJ, Xiao M, Chen R, Cai Z. Metabolic dysfunction of astrocyte: an initiating factor in beta-amyloid pathology? Aging Neurodegener. 2013;1: $7-14$.

42. Shin JY, Park HJ, Kim HN, et al. Mesenchymal stem cells enhance autophagy and increase beta-amyloid clearance in Alzheimer disease models. Autophagy. 2014;10:32-44.

43. Steele JW, Gandy S. Latrepirdine (Dimebon(R)), a potential Alzheimer therapeutic, regulates autophagy and neuropathology in an Alzheimer mouse model. Autophagy. 2013;9:617-618

44. Nixon RA, Wegiel J, Kumar A, et al. Extensive involvement of autophagy in Alzheimer disease: an immuno-electron microscopy study. J Neuropathol Exp Neurol. 2005;64:113-122.

45. Jaeger PA, Wyss-Coray T. Beclin 1 complex in autophagy and Alzheimer disease. Arch Neurol. 2010;67:1181-1184.

46. Ling D, Salvaterra PM. A central role for autophagy in Alzheimer-type neurodegeneration. Autophagy. 2009;5:738-740.

47. Torres M, Jimenez S, Sanchez-Varo R, et al. Defective lysosomal proteolysis and axonal transport are early pathogenic events that worsen with age leading to increased APP metabolism and synaptic Abeta in transgenic APP/PS1 hippocampus. Mol Neurodegener. 2012;7:59. 
48. Ghavami S, Shojaei S, Yeganeh B, et al. Autophagy and apoptosis dysfunction in neurodegenerative disorders. Prog Neurobiol. 2014;112: 24-49.

49. Kuperstein F, Brand A, Yavin E. Amyloid Abeta1-40 preconditions nonapoptotic signals in vivo and protects fetal rat brain from intrauterine ischemic stress. J Neurochem. 2004;91:965-974.

50. Ansari N, Khodagholi F. Molecular mechanism aspect of ER stress in Alzheimer's disease: current approaches and future strategies. Curr Drug Targets. 2013;14:114-122.

51. Vagnoni A, Glennon EB, Perkinton MS, Gray EH, Noble W, Miller CC. Loss of c-Jun N-terminal kinase-interacting protein-1 does not affect axonal transport of the amyloid precursor protein or $\mathrm{A} \beta$ production. Hum Mol Genet. 2013;22:4646-4652.

52. Vagnoni A, Perkinton MS, Gray EH, Francis PT, Noble W, Miller CC. Calsyntenin-1 mediates axonal transport of the amyloid precursor protein and regulates A $\beta$ production. Hum Mol Genet. 2012;21: 2845-2854.

53. Vossel KA, Zhang K, Brodbeck J, et al. Tau reduction prevents Abetainduced defects in axonal transport. Science. 2010;330:198.

54. Winton MJ, Lee EB, Sun E, et al. Intraneuronal APP, not free A $\beta$ peptides in 3xTg-AD mice: implications for tau versus $A \beta$-mediated Alzheimer neurodegeneration. J Neurosci. 2011;31:7691-7699.

55. George AJ, Holsinger RM, McLean CA, et al. APP intracellular domain is increased and soluble Abeta is reduced with diet-induced hypercholesterolemia in a transgenic mouse model of Alzheimer disease. Neurobiol Dis. 2004;16:124-132.

56. Son SM, Song H, Byun J, et al. Accumulation of autophagosomes contributes to enhanced amyloidogenic APP processing under insulinresistant conditions. Autophagy. 2012;8:1842-1844.
57. Makioka K, Yamazaki T, Kakuda S, Okamoto K. Variations in the effects on synthesis of amyloid beta protein in modulated autophagic conditions. Neurol Res. 2009;31:959-968.

58. Tung YT, Wang BJ, Hu MK, et al. Autophagy: a double-edged sword in Alzheimer's disease. J Biosci. 2012;37:157-165.

59. Tamboli IY, Hampel H, Tien NT, et al. Sphingolipid storage affects autophagic metabolism of the amyloid precursor protein and promotes Abeta generation. J Neurosci. 2011;31:1837-1849.

60. Ulamek-Koziol M, Furmaga-Jablonska W, Januszewski S, et al. Neuronal autophagy: self-eating or self-cannibalism in Alzheimer's disease. Neurochem Res. 2013;38:1769-1773.

61. Chun YS, Park Y, Oh HG, et al. O-GlcNAcylation promotes non-amyloidogenic processing of amyloid-beta protein precursor via inhibition of endocytosis from the plasma membrane. J Alzheimers Dis. 2015; 44(1):261-275.

62. Hung SY, Huang WP, Liou HC, Fu WM. Autophagy protects neuron from Abeta-induced cytotoxicity. Autophagy. 2009;5:502-510.

63. Rajadas J, Sun W, Li H, et al. Enhanced $A \beta(1-40)$ production in endothelial cells stimulated with fibrillar $\mathrm{A} \beta(1-42)$. PLoS One. 2013;8: e58194.

64. Cecarini V, Bonfili L, Cuccioloni M, et al. Wild type and mutant amyloid precursor proteins influence downstream effects of proteasome and autophagy inhibition. Biochim Biophys Acta. 2014;1842:127-134.

65. Li W, Poteet E, Xie L, Liu R, Wen Y, Yang SH. Regulation of matrix metalloproteinase 2 by oligomeric amyloid beta protein. Brain Res. 2011; 1387:141-148.

66. Knobloch M, Konietzko U, Krebs DC, Nitsch RM. Intracellular Abeta and cognitive deficits precede beta-amyloid deposition in transgenic arcAbeta mice. Neurobiol Aging. 2007;28:1297-1306.
Neuropsychiatric Disease and Treatment

\section{Publish your work in this journal}

Neuropsychiatric Disease and Treatment is an international, peerreviewed journal of clinical therapeutics and pharmacology focusing on concise rapid reporting of clinical or pre-clinical studies on a range of neuropsychiatric and neurological disorders. This journal is indexed on PubMed Central, the 'PsycINFO' database and CAS,

\section{Dovepress}

and is the official journal of The International Neuropsychiatric Association (INA). The manuscript management system is completely online and includes a very quick and fair peer-review system, which is all easy to use. Visit http://www.dovepress.com/testimonials.php to read real quotes from published authors. 